University of New Hampshire

University of New Hampshire Scholars' Repository

Space Science Center

Institute for the Study of Earth, Oceans, and

Space (EOS)

6-13-2006

\title{
The Advanced Compton Telescope
}

\author{
S E. Boggs \\ University of California - Berkeley \\ J Kurfess \\ Naval Research Laboratory \\ James M. Ryan \\ University of New Hampshire, James.Ryan@unh.edu \\ Elena Aprile \\ Columbia University \\ Neil Gehrels \\ Goddard Space Flight Ctr \\ Follow this and additional works at: https://scholars.unh.edu/ssc \\ See next page for additional authors \\ Part of the Astrophysics and Astronomy Commons
}

\section{Recommended Citation}

Steven Boggs ; James Kurfess ; James Ryan ; Elena Aprile ; Neil Gehrels ; Marc Kippen ; Marc Leising ; Uwe Oberlack ; Cornelia Wunderer ; Allen Zych ; Peter Bloser ; Michael Harris ; Andrew Hoover ; Alexei Klimenk ; Dan Kocevski ; Mark McConnell ; Peter Milne ; Elena I. Novikova ; Bernard Phlips ; Mark Polsen ; Steven Sturner ; Derek Tournear ; Georg Weidenspointner ; Eric Wulf ; Andreas Zoglauer ; Matthew Baring ; John Beacom ; Lars Bildsten ; Charles Dermer ; Dieter Hartmann ; Margarita Hernanz ; David Smith and Sumner Starrfield "The Advanced Compton Telescope", Proc. SPIE 6266, Space Telescopes and Instrumentation II: Ultraviolet to Gamma Ray, 626624 (June 13, 2006); doi:10.1117/12.670605; http://dx.doi.org/10.1117/12.670605

This Conference Proceeding is brought to you for free and open access by the Institute for the Study of Earth, Oceans, and Space (EOS) at University of New Hampshire Scholars' Repository. It has been accepted for inclusion in Space Science Center by an authorized administrator of University of New Hampshire Scholars' Repository. For more information, please contact Scholarly.Communication@unh.edu. 


\section{Authors}

S E. Boggs, J Kurfess, James M. Ryan, Elena Aprile, Neil Gehrels, R M. Kippen, Marc Leising, U Oberlack, Cornelia B. Wunderer, Allen Zych, Peter F. Bloser, M Harris, A Hoover, Alexei Klimenk, Dan Kocevski, Mark L. McConnell, Peter Milne, E I. Novikova, B F. Phlips, Mark Polsen, Steven Sturner, Derek Tournear, G Weidenspointer, Eric Wulf, A Zoglauer, Matthew Baring, John Beacom, Lars Bildsten, Charles Dermer, Dieter H. Hartman, Margarita Hernanz, David Smith, and Sumner Starrfield 


\title{
The Advanced Compton Telescope
}

Steven Boggs ${ }^{\mathrm{a}}$, James Kurfess ${ }^{\mathrm{b}}$, James Ryan ${ }^{\mathrm{c}}$, Elena Aprile ${ }^{\mathrm{d}}$, Neil Gehrels ${ }^{\mathrm{e}}$, Marc Kippen ${ }^{\mathrm{f}}$, Mark Leising $^{\mathrm{g}}$, Uwe Oberlack ${ }^{\mathrm{h}}$, Cornelia Wunderer ${ }^{\mathrm{a}}$, Allen Zych ${ }^{\mathrm{i}}$, Peter Bloser ${ }^{\mathrm{c}}$, Michael Harris ${ }^{\mathrm{j}}$, Andrew Hoover $^{f}$, Alexei Klimenk ${ }^{\mathrm{f}}$, Dan Kocevski ${ }^{\mathrm{h}}$, Mark McConnell ${ }^{3}$, Peter Milne ${ }^{\mathrm{k}}$, Elena I. Novikova ${ }^{\mathrm{b}}$, Bernard Phlipss ${ }^{\mathrm{b}}$, Mark Polsen ${ }^{\mathrm{i}}$, Steven Sturner ${ }^{\mathrm{e}}$, Derek Tournear ${ }^{\mathrm{f}}$, Georg Weidenspointner ${ }^{\mathrm{j}}$, Eric Wulf $^{\mathrm{b}}$, Andreas Zoglauer ${ }^{\mathrm{a}}$, Matthew Baring ${ }^{\mathrm{h}}$, John Beacom ${ }^{1}$, Lars Bildsten ${ }^{\mathrm{m}}$, Charles Dermer ${ }^{\mathrm{b}}$, Dieter Hartmann ${ }^{\mathrm{g}}$, Margarita Hernanz ${ }^{\mathrm{n}}$, David Smith $^{\mathrm{o}}$, Sumner Starrfield ${ }^{\mathrm{p}}$, for the larger ACT collaboration

${ }^{\mathrm{a}}$ Space Sciences Laboratory, University of California, Berkeley, CA USA 94720-7450; ${ }^{\text {NNaval }}$ Research Laboratory; ${ }^{\mathrm{c} U n i v e r s i t y ~ o f ~ N e w ~ H a m p s h i r e ; ~}{ }^{\mathrm{d}}$ Columbia University; ${ }^{\mathrm{e}}$ Goddard Space Flight Center; ${ }^{\mathrm{f}}$ Los Alamos National Laboratory; ${ }^{\mathrm{g}}$ Clemson University; ${ }^{\mathrm{h}}$ Rice University; ${ }^{\mathrm{i}}$ University of California, Riverside; ${ }^{\mathrm{j}} \mathrm{CESR}$, France; ${ }^{\mathrm{k}}$ Arizona State University; ${ }^{\mathrm{l} O h i o}$ State University; ${ }^{\mathrm{m}}$ University of Califonia, Santa Barbara; ${ }^{\mathrm{n}}$ IEEC-CSIC, Spain; ${ }^{\circ}$ University of California, Santa Cruz; ${ }^{\mathrm{p}}$ University of Arizona, Tucson

\begin{abstract}
The Advanced Compton Telescope (ACT), the next major step in gamma-ray astronomy, will probe the fires where chemical elements are formed by enabling high-resolution spectroscopy of nuclear emission from supernova explosions. During the past two years, our collaboration has been undertaking a NASA mission concept study for ACT. This study was designed to (1) transform the key scientific objectives into specific instrument requirements, (2) to identify the most promising technologies to meet those requirements, and (3) to design a viable mission concept for this instrument. We present the results of this study, including scientific goals and expected performance, mission design, and technology recommendations.
\end{abstract}

Keywords: High energy astrophysics; x-ray/gamma-ray detectors; satellite missions; gamma-ray astronomy; gamma-ray spectrometry; gamma-ray imaging

\section{INTRODUCTION}

Since the gravitational collapse of matter into stars and galaxies a few hundred thousand years after the Big Bang, much of the visible matter in the Universe has been through the slow but spectacular lifecycle of matter: stellar formation and evolution ending in novae or supernovae, with the ejection of heavy nuclei back into the galaxy to seed a new generation of stars. The unstable balance between gravitational and nuclear forces produces a cycle in which the death of stars leads to the birth of others, maintaining a rich, dynamic story of life, death, and rebirth on the galactic stages of our Universe.

Nuclear gamma-ray astrophysics is the study of emission from radioactive nuclei as tracers of this cycle of creation. Nuclear decays are "fingerprints" of the isotopes, and the gamma-rays emitted characterize their quantities, speeds, and depths in their environments. The unstable nuclei provide a direct means of quantifying the underlying processes of nuclear burning in supernovae, novae, and hydrostatic stars. They carry unique information about the otherwise hidden, extreme conditions under which they were produced. These nuclei allow us to see to the very core of a supernova explosion, revealing critical information about the underlying nuclear ignition, structure, and dynamics of these events that affect the evolution of our Galaxy.

Because of this potential, the Advanced Compton Telescope (ACT) has been identified as the next major step in gamma-ray astronomy in NASA's roadmap. Its main goal is to probe the nuclear fires creating the chemical elements. For example, thermonuclear supernovae (SN Ia) are used as standard candles across the Universe, yet even those near us are poorly understood. ACT will detect and measure nuclear species produced in those explosions $\left({ }^{56} \mathrm{Ni},{ }^{56} \mathrm{Co}\right)$, 
providing otherwise unattainable information on the dynamics of SN nuclear burning. Supernovae, novae, and stellar winds populate our Galaxy with fresh nuclei. ACT will measure the radioactive gamma-ray and positron emitters among them across the entire Milky Way, mapping our galaxy in a broad range of nuclear line emissions (Fig. 1) from radioactive decays $\left({ }^{22} \mathrm{Na},{ }^{26} \mathrm{Al},{ }^{44} \mathrm{Ti},{ }^{60} \mathrm{Fe}\right)$, nuclear de-excitations $\left({ }^{12} \mathrm{C},{ }^{16} \mathrm{O},{ }^{56} \mathrm{Fe}\right)$ and $\mathrm{e}^{+} \mathrm{e}^{-}$annihilations.

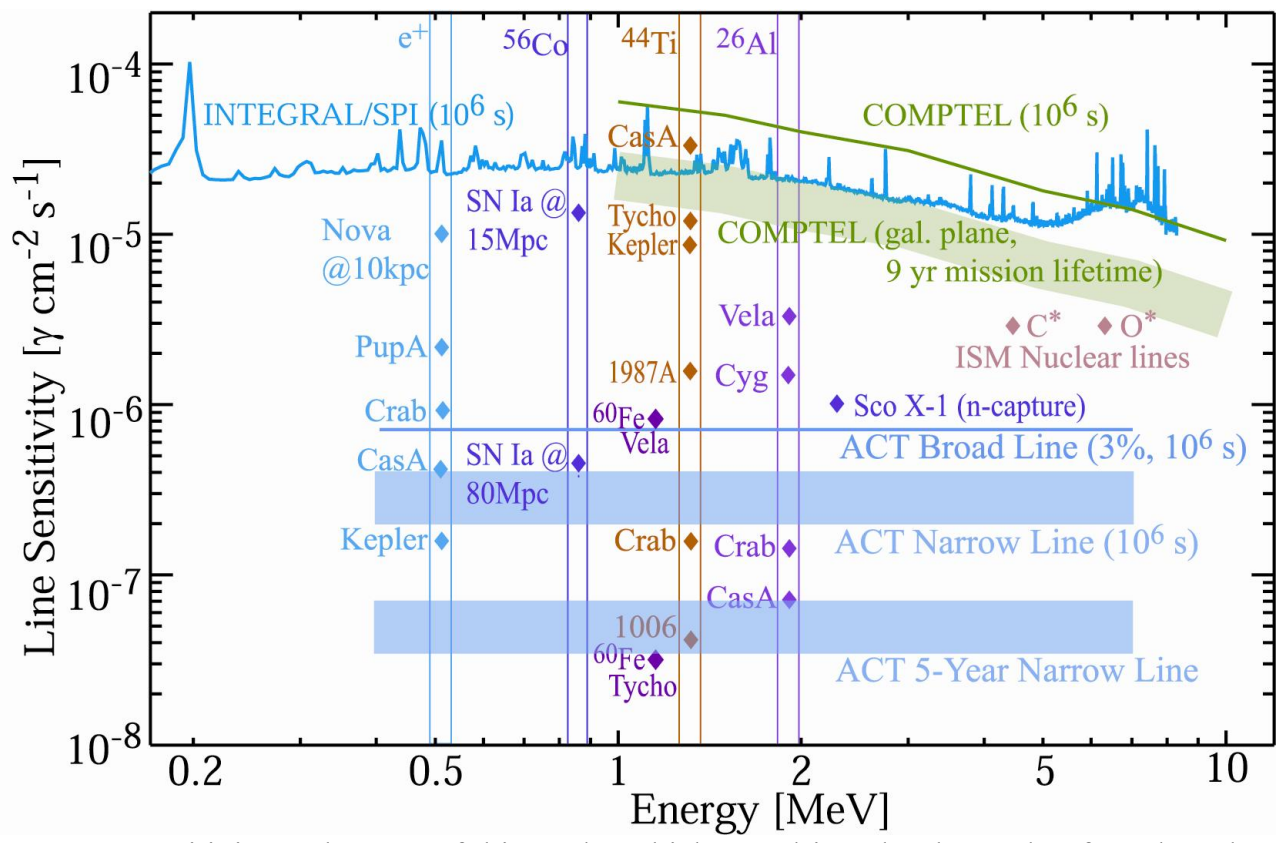

Fig. 1. The ACT target sensitivity at the start of this study, which was driven by the study of $3 \%$-broadened $0.847-\mathrm{MeV}$ $56 \mathrm{Co}$ emission from SNe Ia. The ACT baseline already comes close to this goal, achieving the sensitivity required to systematically study SN Ia, while also achieving major improvements in all-sky sensitivity to narrow lines, $\gamma$-ray emission from compact objects and GRBs, and novel sensitivity to polarization.

As additional objectives (Table 1), gamma-rays from accretion of matter onto galactic compact objects and massive black holes in AGN will test accretion disk and jet models and probe relativistic plasmas. Gamma-ray polarization will be used to study the emission processes in GRBs, pulsars, AGN, and solar flares-opening a new dimension in diagnostic phase space. The origins of the diffuse cosmic $\mathrm{MeV}$ background will be identified.

Previous $\gamma$-ray missions began to address this science, but the key to real advancement is a dramatic improvement in sensitivity. The optimal approach for achieving this improvement is an imaging Compton telescope using advanced detector technology. The technology enabling ACT is the development of 3-D position-sensitive detectors with excellent energy resolution. They resolve interaction sites and energies as photons Compton scatter throughout the instrument, providing a powerful new tool for background rejection, Compton imaging, and polarization studies. The excellent position resolution of these detectors facilitates compact Compton telescopes, increasing the detection efficiency by up to two orders of magnitude over COMPTEL on CGRO [38]. The combination of improved efficiency and improved background rejection will yield $50 \times$ better sensitivity than any previous or current $\gamma$-ray spectrometer or imager. The large FoV achievable with compact designs is also a major improvement, enabling the discovery and monitoring of transient sources, and long exposures of steady-state sources. We restricted this study to instruments designed to attack the SN Ia problem, as opposed to broad MeV scientific objectives. We made this tough choice despite the demonstrated success of multi-instrument missions, e.g., CGRO.

During the course of the ACT concept study, our team participated in two one-week studies at the NASA/Goddard Integrated Design Capability laboratories in order to develop a viable mission concept, and to identify the key technology requirements for the mission. The first study was a one-week run through the Instrument Synthesis \& Analysis Laboratory (ISAL) in September 2004. The primary goals of the ISAL study were to develop a more detailed science instrument model, identify its technical requirements (mass, power, telemetry), and identify the key requirements placed on the mission architecture. The second study was a 1-week run through the Integrated Mission 
Design Center (IMDC) in November 2004, whose primary goal was to take the instrument requirements from the ISAL run and develop an integrated mission architecture package for ACT. The primary finding of our mission design studies is that all of the primary mission architecture requirements are achievable with technologies readily available for launch as early as 2015. The mission and instrument requirements that emerged from this concept study were primarily derived in these ISAL and IMDC runs.

\section{SCIENCE OVERVIEW}

The unique and substantial astrophysical information carried by $\gamma$-ray photons with energies near one $\mathrm{MeV}$ has long been clear. Nuclear $\gamma$-ray line transition energies are practically unique, identifying individual isotopes. In the case of nuclear levels populated by radioactive decay, we also infer the ages of these isotopes, to within a few half-lives. At the electron rest-mass energy $(0.511 \mathrm{MeV})$ the annihilation of electron-positron pairs can be studied in numerous highenergy sources, and the onset of relativistic effects in photon production and interaction processes produces distinctive spectral shapes. Gamma-ray photons are highly penetrating, easily traversing entire galaxies and escaping from supernova ejecta on timescales of weeks. Gamma rays provide probing diagnostics of many astrophysical sources, in some cases the only accessible means of understanding the physics of those sources. The greatest challenge for $\gamma$-ray astronomy has always been small photon fluxes and large backgrounds, so instrument sensitivities are paramount.

ACT is driven in its instrumental performance by some of the most pressing astrophysical questions of our age, the observations of distant Type Ia supernovae and their role in the production of the most abundant heavy element, Iron. The understanding of SNe Ia explosion physics is of critical importance for cosmology, astrophysics, and nucleosynthesis, and places the most stringent sensitivity requirements on ACT (Fig. 1). Therefore, we spent substantial effort studying this requirement. We believe this mission must achieve the Type Ia supernovae objectives, thus the discussion and recommendations that follow are couched in the context of this overarching objective. While most of the other science objectives will follow naturally from the ACT improvements in line and continuum sensitivity (Table 1), some secondary objectives could potentially suffer from this focus.

Table 1. ACT source numbers vs. COMPTEL.

\begin{tabular}{|l|c|c|}
\hline Sources (5yr) & COMPTEL & ACT \\
\hline Supernovae & 1 & $100-200$ \\
\hline AGN \& Blazars & 15 & $200-500$ \\
\hline Galactic & 23 & $300-500$ \\
\hline GRBs & 31 & $1000-1500$ \\
\hline Novae & 0 & $25-50$ \\
\hline
\end{tabular}

The formation of stars and planets, and the development of the chemistry of life, can be understood only in the context of the creation and evolution of the elements. The nucleosynthesis products are interesting as signposts of our origins, as well as diagnostics of the poorly understood supernova explosion mechanisms. ACT's direct observations of nucleosynthesis products in diverse environments, including individual new supernovae as well as supernova remnants, will yield deep new insights.

Supernovae have synthesized most of the elements heavier than He and supplied much of the energy input to the interstellar medium. Most of the newly created nuclei are indistinguishable from older nuclei that predate the star or were produced in hydrostatic phases; however, radioactive species among the ejecta serve as definitive tracers of recent nuclear processing. Some nuclei, such as the atomic mass A=56 chain, provide much of the power for the optical displays. The $\gamma$-ray lines from the decay of these nuclei reveal the location of the radioactivity within the ejecta through the time-dependence resulting from the unfolding of the attenuating material, and the ejection velocities of various layers from the line Doppler shifts. Spectroscopy and lightcurve measurements of these $\gamma$-ray lines allow direct measurement of the underlying explosion physics and dynamics.

$\mathrm{SNe}$ Ia, the thermonuclear explosions of degenerate white dwarfs, are profoundly radioactive events. As much as onehalf of the white dwarf mass is fused to ${ }^{56} \mathrm{Ni}\left(\mathrm{t}_{\text {half }}=6.1 \mathrm{~d}\right)$. After a short time, it is the decay of this nucleus and its daughter, ${ }^{56} \mathrm{Co}\left(t_{\text {half }}=77 \mathrm{~d}\right)$, which power the entire visible display of the supernova. Most of this power, however, is 
emitted in the form of $\gamma$-ray lines, some of which begin to escape after several days. These $\gamma$-rays are the most direct diagnostic of the dominant processes in the nuclear burning and explosion.

Despite extensive study of SNe Ia, and their use in cosmology, major fundamental questions about these explosions remain unanswered. For example, we do not know their progenitor systems [8]. Almost certainly they occur in binary systems, but the nature of the companions, whether normal stars, white dwarfs, or some of each, is unknown. Thermonuclear supernovae are also grand experiments in reactive hydrodynamical flows. We do not understand how the nuclear flame propagates, how it proceeds as fast as it does, or how and when it turns into a shock (or even whether, though there is some evidence that it does) and propagates supersonically [16]. We do not know to what extent instabilities break spherical symmetry, or whether their effects are wiped out by subsequent burning if they do. We do not fully understand the empirical correction to their magnitudes that allows them to be used as standard candles for measuring the geometry of our Universe.

Nuclear $\gamma$-ray lines from SNe Ia hold the key to solving these mysteries. ACT has three primary science goals for studying SNe Ia, and these goals are the primary drivers of ACT's instrumental performance requirements (broad-line sensitivity, spectral resolution, FoV).

ACT's goals are aggressive:

1. Standard Candles. Characterize the ${ }^{56} \mathrm{Ni}$ production distribution for SNe Ia, and correlate with the optical lightcurves to determine the relationship between empirical absolute magnitude corrections and ${ }^{56} \mathrm{Ni}$ production. Requirements: measurement of ${ }^{56} \mathrm{Ni}$ production in $>100$ SNe Ia at $>5 \sigma$ levels.

2. Explosion Physics. Clarify the nuclear flame propagation and ejecta mass and kinematics for a key handful of SNe Ia,

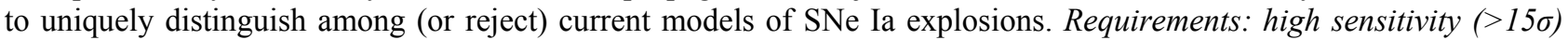
lightcurves and high-resolution spectra $(\Delta E / E<1 \%)$ of several SNe Ia events of each subclass over the primary 5-year survey.

3. SN Ia Rate-Local and Cosmic. Measure the SN Ia rates in the local universe, unbiased by extinction and solar constraints, and the cosmic SNe Ia history. Requirements: all-sky survey of SNe Ia, sensitive to distances beyond the Virgo cluster and measurement of the cosmic $\gamma$-ray background spectrum with sufficient energy resolution to separate the contributions from SNe Ia and AGN

OSSE and COMPTEL detection of ${ }^{56} \mathrm{Co}$ emission, and discrimination between SN explosion scenarios were two of the primary goals of the CGRO mission. However, the two SNe Ia that were observed with CGRO, SNe 1991T and 1998bu, were too distant to conclusively discriminate among dominant explosion scenarios $[27,23,22,15]$.

\subsubsection{Standard Candles: Physics \& Evolution}

Although many fundamental questions remain about SNe Ia, they are still being used as standard candles to measure cosmological distances with dramatic implications $[31,34]$. Though intrinsically variable in their absolute magnitudes, the Phillips relation can be used to correct the measured luminosities, $\Delta \mathrm{m}_{15}(\mathrm{~B})$. This empirical relation, deduced from the width in time of their visible light curves, assumes there is a one to one mapping between duration and luminosity [32]. Whether there is truly one physical parameter of the explosion that determines both is not certain. Whether the same relationship should hold exactly to redshifts approaching unity and beyond, when, for example, the metallicity of the systems was lower, is also not clear [14]. Complete confidence in the accuracy of using SNe Ia to measure such distances awaits better understanding of the explosions themselves.

ACT will allow direct correlation for over $100 \mathrm{SNe}$ Ia between the optical properties and the ${ }^{56} \mathrm{Ni}$ production, which is likely to be the underlying factor in the optical lightcurve variations. For example, Fig. 2 shows a simulated distribution of measured ${ }^{56} \mathrm{Ni}$ production versus the optical magnitude correction, assuming a linear correlation between the two. $\mathrm{ACT}$ is also sensitive to other possible causes of the light curve variation, such as ${ }^{56} \mathrm{Ni}$ distribution in velocity (through spectroscopy), and total ejecta mass (through light curve monitoring), which can be distinguished from the total ${ }^{56} \mathrm{Ni}$ mass explanation. Such measurements will allow us to directly probe the underlying physical mechanisms driving the 
variations in the optical curves. Coupled with ACT's ability to uncover the explosion mechanism and dynamics, a much better understanding of how SNe Ia evolve with redshift will be possible, as well as confidence in their use as standard candles to high $Z$.

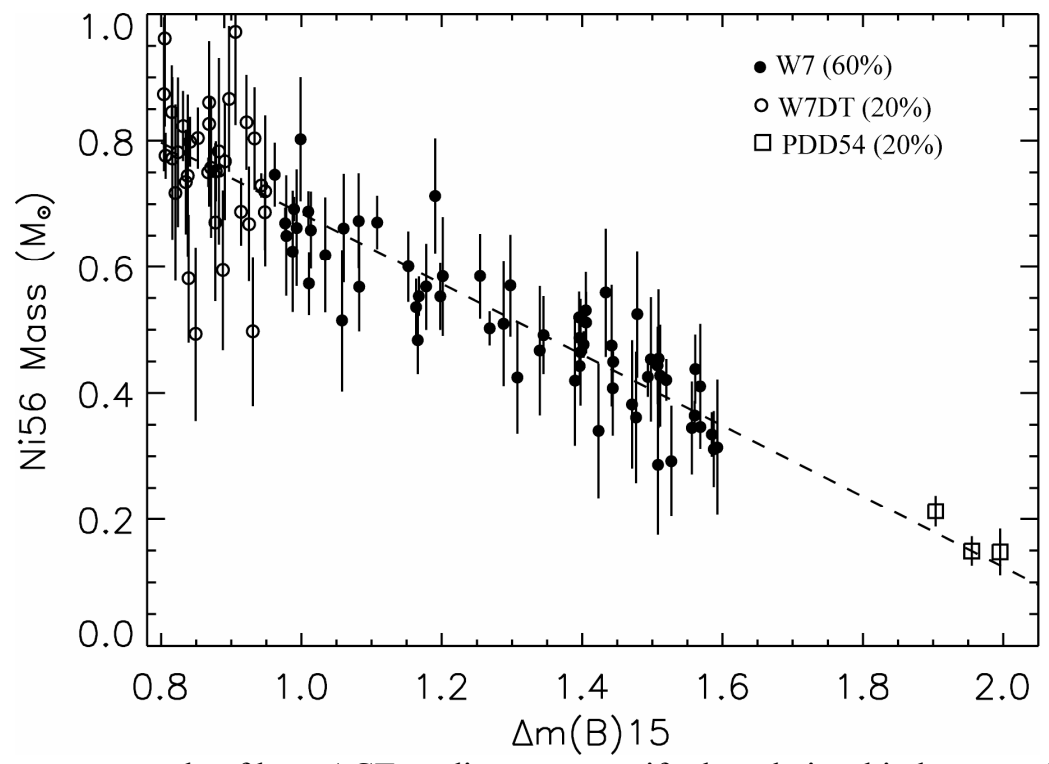

Fig. 2. This figure shows an example of how ACT studies can quantify the relationship between $\mathrm{SNe}$ Ia ${ }^{56} \mathrm{Ni}$ production and the optical lightcurve variations. Shown is a 5-year cumulative measurement of ${ }^{56} \mathrm{Ni}$ from all SNe Ia detected above $5 \sigma$, assuming a ratio of SN explosions of 60:20:20 between normal (W7), superluminous (W7DT), and subluminous (PDD54) SNe Ia. It is assumed for this simulation that the ${ }^{56} \mathrm{Ni}$ mass is correlated with the optical magnitude correction by the relation $\mathrm{M}_{56}=1.24-0.56^{*} \Delta \mathrm{m}_{15}(\mathrm{~B})$ (dashed line).

\subsubsection{Uncovering the Explosion Physics}

Although a variety of SN Ia progenitors have been suggested, three dominant scenarios have emerged. (1) A single CO white dwarf accretes mass from a donor star until it (nearly) reaches the Chandrasekhar mass and suffers a thermonuclear runaway. (2) Two smaller $\mathrm{CO}$ white dwarfs merge to form a single $\mathrm{CO}$ white dwarf of the Chandrasekhar mass surrounded by an envelope, perhaps leading to an off-center thermonuclear runaway. (3) A single, low mass $\mathrm{CO}$ white dwarf accretes and burns hydrogen to form a thick helium shell that suffers helium ignition at its base, triggering a thermonuclear runaway in the $\mathrm{CO}$ white dwarf (sub-Chandrasekhar). Within the first scenario, it has been suggested that the burning front proceeds entirely sub-sonically as a deflagration, or alternatively, that it later accelerates to produce a detonation in the outer, near-surface layers of the exploding white dwarf (referred to as a delayed detonation).

During the 1990's many SNe Ia were well studied optically, and the dominant explosion scenarios were simulated with radiation transport codes. It is the consensus (although not universally accepted), that the sub-Chandrasekhar mass explosions produce optical spectra that do not well match the observations. Generally, sub-Chandrasekhar mass models appear to be inconsistent with normal SNe Ia, but perhaps are able to explain some fraction of the peculiar events. This stance is perhaps too charitable, as the spectra predicted for these events have never been clearly detected from an observed SN Ia. Recent studies of the optical emission from individual SNe Ia find features that tend to favor delayed detonations over deflagrations (e.g., $[35,5])$.

It has long been recognized that the amount and location of the ${ }^{56} \mathrm{Ni}$ in supernova explosions could be deduced from $\gamma$ ray line observations [9], and specifically that the differences between the Chandrasekhar mass explosions (that produce more total ${ }^{56} \mathrm{Ni}$, but bury it under a significant amount of more slowly expanding material) and sub-Chandrasekhar mass explosions (that burn a large fraction of their total mass to ${ }^{56} \mathrm{Ni}$ but produce lower total ${ }^{56} \mathrm{Ni}$ yields than the equivalent $\mathrm{CM}$ explosion) could lead to significant differences in the $\gamma$-ray line flux light curves and line spectra from SNe Ia. 
Moreover, within the Chandrasekhar mass explosion scenarios the near-surface ${ }^{56} \mathrm{Ni}$ produced in the delayed detonation would also substantially alter the $\gamma$-ray properties from a deflagration explosion [18]. There has not been as much effort simulating merger scenarios as there has been in the other explosion scenarios, but indications are that their $\gamma$-ray spectra are intermediate between sub-Chandrasekhar mass models and Chandrasekhar mass models.

In this study, we concentrate on deflagration versus delayed detonation flame propagation, and, to a lesser extent, Chandrasekhar-mass versus low-mass models.

The most stringent performance constraint on ACT is set by its goal to definitively discriminate between deflagrations and delayed detonations for a handful of events during the ACT mission. To achieve this, we must discriminate between these two scenarios to $20 \mathrm{Mpc}$, i.e., roughly the distance within which there is one SN Ia per year. (We adopt that distance for our comparisons.). With peak line fluxes of $\sim 10^{-5} \mathrm{~cm}^{-2} \mathrm{~s}^{-1}$ for even near SNe Ia ( 15 Mpc), which surpasses current detection sensitivities, it is clear that a significant improvement in sensitivity over previous and current missions is required (Fig. 1). We select three models for this investigation: a Chandrasekhar mass deflagration (W7 [28]), a delayed detonation (DD202C [18]), and a sub-Chandrasekhar mass explosion (HED8 [17]). We use the subChandrasekhar mass HED8 in our study rather than the more commonly referenced HED6, because of the larger ${ }^{56} \mathrm{Ni}$ production in HED8. By doing this, we compare three models that are more likely to represent normally-luminous $\mathrm{SNe}$ Ia.

Our requirement is that we distinguish the deflagration model from the delayed detonation model, even if the distance (and therefore absolute line luminosity) is unknown. Clearly, the combined information from optical and $\gamma$-ray studies will be utilized, but we set the goal: ACT must be able to detect the differences in the line profiles and/or light curve shapes alone. This is a difficult problem we have set in front of ACT: DD202C and W7 have similar $\gamma$-ray properties. For the two models we calculate the spectra versus time, do a least squares normalization of the two sets, subtract them, and determine whether we can detect the difference for a given instrument spectral resolution, sensitivity, and observing strategy. Note that these lines are Doppler broadened to 3-5\%, so that broad line sensitivity is (for this objective) the relevant instrumental requirement.

For distinguishing explosion models it is not adequate to set a single sensitivity requirement: instruments with higher spectral resolution can distinguish explosion models better than instruments with poorer spectral resolution, given the same sensitivity. Therefore, we developed curves that show our ability to distinguish explosion models as a function of both the instrumental spectral resolution and broad-line sensitivity. The differences between the models manifest themselves in two primary ways in the $\gamma$-ray data: lightcurves of the emission lines, and the Doppler-broadened profiles of the lines. Therefore, both sensitivity and spectral resolution are required to distinguish the models. These requirements are discussed further in Section $\mathrm{G}$, but typical requirements to clearly distinguish these two models $(5 \sigma)$ at

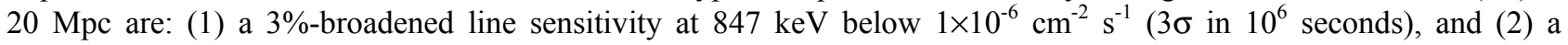
corresponding spectral resolution better than $1 \%$. The poorer the spectral resolution for an instrument, the stricter is the sensitivity requirement to distinguish these models.

\subsubsection{SNe Ia Rates-Local}

ACT will detect a large number of SNe Ia, but it will be the closest few that will most illuminate thermonuclear supernova physics. It is difficult to extrapolate the rates on larger scales to the very local, nonuniform universe, but actual SN Ia discoveries suggest that the rate within $20 \mathrm{Mpc}$ distance is adequate for our goals. Simply taking confirmed SNe Ia in the past ten years, and taking distances of their host galaxies from Tully's (1988) Nearby Galaxies Catalog, gives the cumulative distribution of SNe Ia vs. distance. This sample still suffers from incompleteness (even the brightest SN display a zone of avoidance along the galactic plane), but at least one SN Ia per year closer than $20 \mathrm{Mpc}$ is assured.

ACT will survey the entire sky continuously, uniformly detecting SNe Ia to distances well beyond the Virgo cluster. Its wide FoV and all-sky survey strategy will discover over a hundred supernovae over its 5-year survey, and provide an unbiased, direct determination of the SN Ia rate in this volume - an independent check of SN Ia rates based on optical studies. In many cases ACT will discover relatively nearby SNe Ia before ground-based searches, including some that 
would be otherwise missed completely. Scanning much of the sky frequently and sensitive to nearby $\mathrm{SNe} 10-12$ days pre-maximum, ACT will detect these events with high efficiency.

\subsubsection{SNe Ia Rate-Cosmic Gamma-Ray Background}

As noted originally by Clayton and Silk [10], it should be possible to measure the diffuse cosmic background of $\mathrm{MeV} \gamma$ rays from the integrated SNe Ia in the universe, in addition to individual nearby SNe Ia. The accumulated and redshifted $\gamma$-ray line spectra form a continuum, with its normalization and spectral features probing the rate and redshift evolution of the contributing SN Ia. While both COMPTEL and SMM measured a $\gamma$-ray continuum in the MeV region [44, 43], it was unclear if it could be associated with the expected SN Ia signal $[42,36]$. The normalization of the SN Ia prediction was quite uncertain, and the data were not precise enough to conclusively test for the expected spectral features.

The SN Ia contribution to the MeV cosmic $\gamma$-ray background depends on three basic ingredients: (i) the cosmic star formation rate history, (ii) the progenitor models of SNe Ia, which determine the efficiency and time delay with which newly formed stars produce SN Ia, and (iii) the $\gamma$-ray emission per SN Ia. Recent improvements in the measurements of the star formation rate and cosmological parameters have helped constrain (i). Assuming $\sim 0.6 \mathrm{M}_{\mathrm{sol}}$ of ${ }^{56} \mathrm{Fe}$ per $\mathrm{SN}$ Ia, and the limited SN Ia rate data available now, the SN Ia contribution to the cosmic $\gamma$-ray background falls short of the measured data $[40,1]$.

This means that there is presently no accepted explanation for the origin of the MeV cosmic $\gamma$-ray background. Speculative extrapolations of the backgrounds from active galaxies may account for some of the data. As discussed in Ref. [40], the MeV region is of special importance for constraining models of exotic physics, e.g., dark matter decay or annihilation. In order to make further progress on resolving this mystery, better characterization of the rates and emission from SNe Ia and active galaxies are needed-ACT will provide this information.

The other essential item that ACT will provide is a new and precise measurement of the $\mathrm{MeV}$ cosmic $\gamma$-ray background. The science requirements focus attention on the importance of very good energy resolution (to test for possible spectral features) and control of detector backgrounds (to allow the full-sky diffuse measurement). In addition, good angular resolution $\left(\sim 1^{\circ}\right)$ would enable angular correlations of the data with themselves and other tracers of cosmic structure, much as has been done for the cosmic microwave background data. As shown in Ref. [46], cosmic $\gamma$-ray background from sources that follow the observed clustering of galaxies should produce detectable angular correlation signals; in contrast, the signals expected in some exotic physics models will be purely isotropic. With very good energy and angular resolution, it may be possible to isolate the SN Ia contribution, even if it is small, which would provide an important test of the predictions of SN Ia progenitor and explosion models.

\section{BASELINE SCIENCE INSTRUMENT}

While we investigated in detail a number of different instrument concepts for ACT during this study, there are several design parameters that are nearly universal. The optimal ACT design consists of at least $2 \mathrm{~m}^{2}$ arrays of position-sensitive spectroscopy detectors in order to achieve effective areas of $\sim 1000 \mathrm{~cm}^{2}$. The depth of the sensitive volume for stopping $\mathrm{MeV}$ gamma-rays must be $35-50 \mathrm{~g} / \mathrm{cm}^{2}$, with a weak dependence on the detector materials. Detectors must be reliable, uniform, and radiation hard. All designs require a surrounding charged-particle anticoincidence detector (ACD), composed of thin scintillator plastic, surrounding the detector array to veto cosmic-ray and trapped particle backgrounds. In addition, we have determined that in LEO, the dominant background component for all designs studied is Earth albedo gamma-rays. A massive bismuth germanate (BGO) scintillator shield beneath the detector array in the baseline model helps suppress this background. 


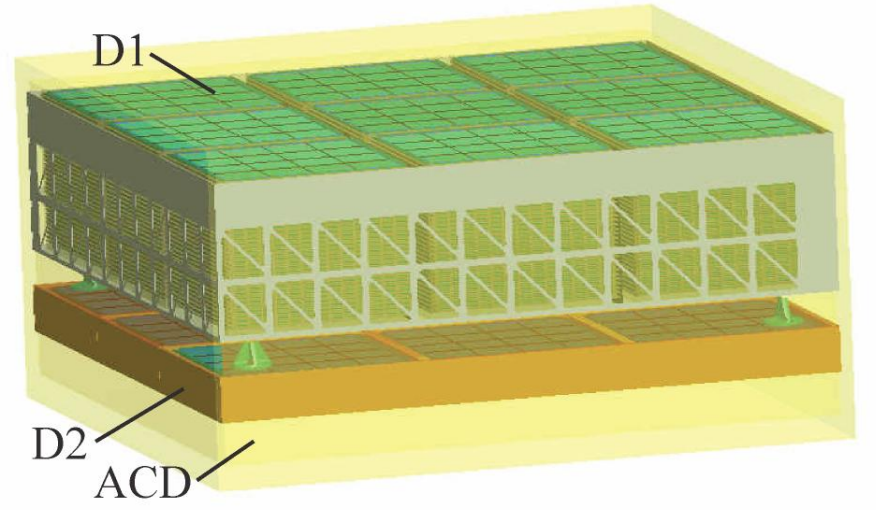

Fig. 3. Baseline ACT design. D1: 27 layers of 2-mm thick Si detectors. D2: 4 layers of 16-mm thick Ge detectors.

Early in the study we selected a "baseline" instrument for the ISAL run that was designed both to be a promising option scientifically, and to encompass a variety of the challenges an ACT instrument might pose. This baseline was subsequently optimized for overall sensitivity, staying within the mission envelope (mass, power size) established in the ISAL run. This optimized baseline concept consists of a hybrid Si-Ge array, consisting of a 27-layer "D1" array of 2$\mathrm{mm}$ thick silicon (low Z) detectors, situated immediately above a 4-layer "D2" array of 16-mm thick germanium (high Z) detectors (Fig. 3). Scientifically, this hybrid design represented a promising choice because it combines the higher intrinsic angular resolution achievable by having a first scatter in the low-Z silicon (less Doppler broadening) with the better stopping power of the high- $Z$ germanium for higher efficiency. Both of these technologies assumed the excellent spatial resolution $\left(<1 \mathrm{~mm}^{3}\right)$ and excellent spectral performance $(\sim 0.2 \%$ at $1 \mathrm{MeV})$ that has been achieved in the laboratory. Neither detector array assumed any electron-tracking or fast-timing capabilities. This hybrid design seemed especially appropriate for the ISAL run because it combined the technical challenges of cooling a large array of Ge detectors to $80 \mathrm{~K}$ with that of powering a large number of readout channels with moderate cooling for the Si detectors $\left(\sim 250,000\right.$ channels, $\left.-30^{\circ} \mathrm{C}\right)$. The instrument's bottom and sides are surrounded by a 4 -cm thick BGO shield.

The ultimate incarnation of ACT will be the result of detailed scientific and technology trades that we have only begun in this concept study. Toward this end, however, we have studied competing technologies that could either augment or supplant those chosen for the baseline design. These include thin Si [29, 19], liquid Xe (LXe) [2-4], CdZnTe [37, 25, 30], $\mathrm{LaBr}$ scintillator [39], and gaseous Xe (GXe) detectors [6, 12, 13]. Each of these detectors possesses capabilities that could potentially improve the performance over the baseline instrument-in terms of electron tracking (thin $\mathrm{Si}$, $\mathrm{GXe}$ ), fast timing ( $\mathrm{LXe}, \mathrm{LaBr}, \mathrm{GXe}$ ), or room-temperature operation ( $\mathrm{CdZnTe}, \mathrm{LaBr}$ ). However, these benefits currently come with a performance hit in other areas including efficiency (thin Si, GXe), spectral resolution (LXe, GXe, $\mathrm{CdZnTe}, \mathrm{LaBr}$ ), and overall power (thin $\mathrm{Si}, \mathrm{GXe}$ ). None of these detector enhancements improved the SNe Ia sensitivity over the ACT baseline (some degraded sensitivity considerably). With the caveat of the limited resources available for this study, and the limited time for optimizing the individual designs, it is still clear that the baseline Si-Ge geometry remains a promising design to pursue for $\mathrm{ACT}$, and most of the alternate designs will have to strive to reach its performance. In addition, we studied two additional instruments composed of all Ge detectors and all thick Si detectors similar to those used in the baseline. The all-Ge instrument is the only design with comparable sensitivity to the $\mathrm{Si}-\mathrm{Ge}$ baseline. Below, we describe this optimized Si-Ge baseline instrument and subsystems, and discuss its performance. 

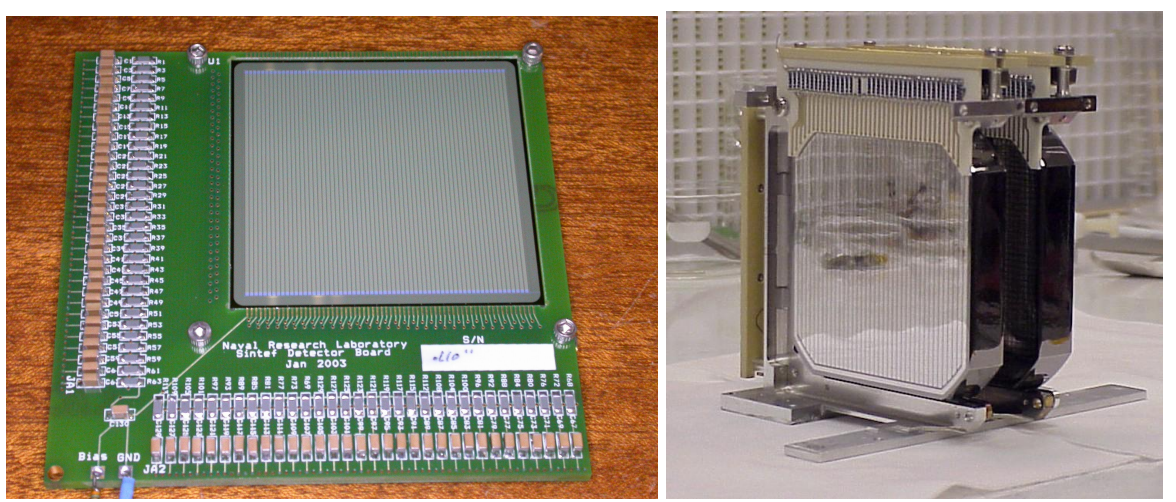

Fig. 4. Detector technologies assumed for the ACT baseline instrument. Left: 2-mm thick Si cross-strip detector [21, 33], Right: 16-mm thick Ge cross-strip detectors [7, 11, 20].

D1 Silicon Detector Array. The low-Z "scattering" array, D1, consists of 27 layers of 2-mm thick double-sided Si crossstrip detectors (Fig. 4). Each layer has 144 detectors, corresponding to 3888 detectors total. Each detector is $10 \times 10 \mathrm{~cm}^{2}$ in area, instrumented with $64 \times 64$ strips $(1.5-\mathrm{mm}$ pitch) and equipped with a $2-\mathrm{mm}$ wide guard ring around the edges. Within one layer, $2 \times 2$ of these individual detectors' strips are connected for a "daisy-chained" readout with 248,832 channels, designed to reduce power consumption and passive material. We assumed a $0.5 \%$ failure rate of these individual electronics channels in our simulation of instrument performance. The Si layers are spaced $10 \mathrm{~mm}$ apart. Each $\mathrm{Si}$ detector is assumed to have $\sim 0.2 \%$ energy resolution at $1 \mathrm{MeV}$, comparable to currently measured laboratory performance $[21,33]$. The Si detectors must be cooled to $-30^{\circ} \mathrm{C}$ in order to achieve their optimal spectral performance. It was determined in the ISAL run that mechanical cooling was the most efficient method for cooling this array (as opposed to passive cooling), and this approach was adopted for the IMDC run. The total active Si mass is $291 \mathrm{~kg}$, with a thickness of $13 \mathrm{~g} / \mathrm{cm}^{2}$.

D2 Germanium Detector Array. The high-Z "absorbing" array, D2, consists of four layers of 16-mm thick Ge cross-strip detectors (Fig. 4). Each layer has 144 detectors, corresponding to 576 detectors total. Each detector is $9.2 \times 9.2 \mathrm{~cm}^{2}$ in area, instrumented with $90 \times 90$ strips $(1.0-\mathrm{mm}$ pitch) and equipped with a 1-mm wide guard ring around the edges. The guard ring is instrumented to provide veto signals. Each strip is read out individually, for a total of 103,680 channels. We assumed $0.5 \%$ failure rate of these individual electronics channels in our simulation of instrument performance. The Ge layers are spaced $25 \mathrm{~mm}$ apart, center-to-center. Each Ge detector is assumed to have $\sim 0.2 \%$ energy resolution at 1 $\mathrm{MeV}$, and $0.4 \mathrm{~mm}$ depth resolution-comparable to currently measured laboratory performance $[7,11,20]$. The Ge detectors must be cooled to $80 \mathrm{~K}$ in order to achieve their optimal spectral performance. It was determined in the ISAL run that a Turbo-Brayton cryocooler would be the most efficient method for cooling this array, which was the assumed method for the IMDC run. The total active Ge mass is $415 \mathrm{~kg}$, with a thickness of $34 \mathrm{~g} / \mathrm{cm}^{2}$.

Readout Electronics. ACT will require the design and development of low-power application specific integrated circuits (ASICs) to read out the hundreds of thousands of channels in the instrument. These ASICs must have low power, large dynamic range, low-noise to achieve the required energy resolution associated with these semiconductor detectors, and $\sim 10 \mathrm{~ns}$ time resolution to provide the depth resolution within the Ge detectors. The ISAL assumed $1 \mathrm{~mW} / \mathrm{chn}$ power consumption for these ASICs, based on optimistic progress in performance and power. Development of low-power ASICs was identified as one of the primary technology development priorities for ACT.

Given the large volume detectors, and high event rates, of ACT, a coincidence time resolution of $150 \mathrm{~ns}$ or better is required for discriminating Compton scatter events from chance coincidences. This coincidence resolution will keep random coincidences from dominating the telemetry stream, while even further discrimination based on Compton kinematics can be performed to reject remaining random coincidences through ground-based processing

Cryogenics. The ACT baseline instrument requires two separate cryogenic systems for the two detector arrays: a system that goes to $80 \mathrm{~K}$ for the Ge (D2) detectors, and a system at $-30^{\circ} \mathrm{C}$ for the Si (D1) detectors. Both the ISAL and the IMDC teams recommended that these be separate cryogenic systems given the very different cooling requirements of the two detector types. 
For the Ge detector array at $80 \mathrm{~K}$, assuming an internal power dissipation of $1 \mathrm{~mW} / \mathrm{chn}$ within the cryostat at an intermediate temperature of $125 \mathrm{~K}$, the heat lift requirements for the entire array are $61 \mathrm{~W}$. The ISAL team identified the Turbo Brayton mechanical cooler used on HST NICMOS [47], combined with cooling loops, as the best candidate technology to fit these requirements. This crycooler offers the advantage of essentially zero vibrations, and it is staightforward to scale to larger sizes.

For the Si detector array at $-30^{\circ} \mathrm{C}$, assuming an internal power dissipation of $1 \mathrm{~mW} / \mathrm{chn}$ at the detector temperature, the total heat lift requirements for the entire array are $253 \mathrm{~W}$. The ISAL and IMDC teams identified the commercial Sunpower cryocoolers currently flying on the RHESSI mission [24] as a good match for this array.

BGO Shield. Two of the largest sources of background in an unshielded soft gamma-ray detector operating in low-earth orbit are albedo photons and secondary photons from interactions of ambient photons, leptons and hadrons in the spacecraft (S/C). For a zenith-pointing instrument, both of these background components can be significantly reduced by covering the bottom and sides of the detector with an active shield that provides enough stopping power for most gamma-rays to interact.

For this purpose, high-density, high-Z scintillators such as BGO are the material of choice. The optimum thickness for this shield has to be determined in a tradeoff study between (photon) stopping power on one hand and weight and neutron activation on the other hand; 3-4 cm appear a reasonable guess based on simulations and prior experience with INTEGRAL/SPI [41].

A 4-cm BGO shield for ACT comprises $\sim 1000 \mathrm{~kg}$ of BGO, a 3-cm shield $\sim 750 \mathrm{~kg}$. This is on the same order as the BGO shield of INTEGRAL/SPI $(517 \mathrm{~kg})$, with much less complexity in the required shapes of BGO crystals and consequently higher crystal light yields and less passive structural mass. We envision a redundant readout of each of 100-200 pieces of BGO with two separate PMTs or photodiodes with individually adjustable gains and thresholds for maximum adaptability of the system. The BGO must provide a prompt $(<0.5 \mu \mathrm{s})$ veto signal to the ACT trigger electronics whenever an interaction occurs above the trigger threshold of $\sim 75 \mathrm{keV}$.

Anti-Coincidence Detector. In low earth orbit, the intensity of particle radiation is many orders of magnitude larger than the astrophysical gamma-ray flux. Thus, the anti-coincidence detector (ACD), which provides an electronic shield, is an integral part of the ACT telescope design. The ACD must provide a prompt $(<0.5 \mu \mathrm{s})$ veto signal to the ACT trigger electronics whenever a charged particle crosses the active volume of the telescope, and have a probability of missing a charged particle below $10^{-6}$. These requirements are similar to those of the GLAST ACD [26], and a similar modular ACD design is envisioned for ACT.

Light from each of $\sim 100$ scintillator tiles will be collected by two independent sets of wavelength shifting fibers and viewed by two separate PMTs with individually adjustable gains and thresholds, providing two-fold readout redundancy as well as a high level of adaptability.

On-board Processing. In the baseline approach developed at the ISAL and IMDC runs, the ACT science instrument will require an internally-redundant science processor to perform data filtering before passing on the data to the main $\mathrm{S} / \mathrm{C}$ computer, as well as two (redundant) digital signal processing (DSP) boards to perform real-time on-board analysis for transient events.

For data handling, the science processor will be performing simple event-level filtering to identify likely Compton events and package them for transfer to the main $\mathrm{S} / \mathrm{C}$ computer. This filtering removes the large number of lowerenergy events in the instrument, though current estimates suggest that these photons would not overwhelm the telemetry stream. The ISAL and IMDC runs identified a PowerPC 750 processor as suitable to fulfill the ACT requirements.

The DSP will be performing a real-time analysis of transient events, with the goal of localizing gamma-ray bursts within a few seconds for rapid telemetry of coordinates to the ground. The IMDC study recommended that this processing should be implemented as a hardware or firmware function by addition of the DSPs to the science processor. 
Instrument Parameters-Mass, Power, Size. We designed the optimized Si-Ge baseline instrument to conform to the ISAL and IMDC findings in terms of available lifting power, size constraints, and power requirements.

The Si-Ge instrument itself, including support structure and co-located electronics as well as the plastic anticoincidence, weighs $831 \mathrm{~kg}$; the lower-level electronics boxes and cryocooler add another $142 \mathrm{~kg}$. The 4-cm thick BGO shield consists of $1081 \mathrm{~kg} \mathrm{BGO}$ and $29 \mathrm{~kg}$ support structure. The main instrument plus all electronics and support have a total mass of $1002 \mathrm{~kg}$; including the 30\% margin assumed at IMDC brings this to $1303 \mathrm{~kg}$. For the BGO itself, no mass margin need be assumed since the material is well known. Thus the total instrument mass with contingency is $2384 \mathrm{~kg}$. This baseline ACT requires $1830 \mathrm{~W}$ for instrument readout and cooling, plus high-level electronics, heaters, and LV power supplies (total $270 \mathrm{~W}$ according to ISAL) and BGO veto readout $(\sim 50 \mathrm{~W})$ for an instrument total of $2150 \mathrm{~W}-$ well within the $2500 \mathrm{~W}$ envelope (including margins) set at the IMDC. The instrument-including BGO and ACD shields-fits within a $150 \times 150 \times 35 \mathrm{~cm}^{3}$ envelope, fitting the $\mathrm{S} / \mathrm{C}$ bus and Delta IV shroud as studied at the IMDC.

The ISAL baselined ASICs with $1 \mathrm{~mW} / \mathrm{channel}$, feeling this was reasonable but pointing out these needed to be developed. ISAL recommended active over passive cooling to $-30^{\circ} \mathrm{C}$ for the Si detectors. Additional areas of concern were the demands on telemetry (rough assumptions yielded $25000 \mathrm{evts} / \mathrm{s}$ resulting in $60 \mathrm{Mbit} / \mathrm{s}$ continuous telemetry stream) and power (with power driven by both cooling and the large number of channels), as well as the desire for a low-inclination orbit carrying a large lift penalty. All the ISAL areas of concern were addressed in the IMDC run, as presented in the Section 2.a.

Table 2. ACT baseline instrument performance.

\begin{tabular}{|l|l|}
\hline Energy range & $0.2-10 \mathrm{MeV}$ \\
\hline Spectral resolution & $0.2-1 \%$ \\
\hline Field of view (FoV) & $25 \%$ sky, $90^{\circ} \mathrm{FWHM}$ \\
\hline Sky coverage & $80 \%$ per orbit \\
\hline Angular resolution & $\sim 1^{\circ}$ \\
\hline Point source localization & 5 \\
\hline Detector area, depth & $12,000 \mathrm{~cm}^{2}, 47 \mathrm{~g} / \mathrm{cm}^{2}$ \\
\hline Effective area & $\sim 1000 \mathrm{~cm}^{2}$ \\
\hline $3 \%$ broad line sensitivity & $1.2 \times 10^{-6} \gamma \mathrm{cm}^{-2} \mathrm{~s}^{-1}$ \\
\hline Narrow line sensitivity & $5 \times 10^{-7} \gamma \mathrm{cm}^{-2} \mathrm{~s}^{-1}$ \\
\hline Continuum sensitivity & $(1 / \mathrm{E}) \times 10^{-5} \mathrm{~cm}^{-2} \mathrm{~s}^{-1} \mathrm{MeV}^{-1}$ \\
\hline GRB fluence sensitivity & $3 \times 10^{-8} \mathrm{erg} \mathrm{cm}$ \\
\hline Data mode & every event to ground \\
\hline
\end{tabular}

\section{INSTRUMENT PERFORMANCE}

Simulations of the optimized Si-Ge baseline instrument with BGO shield in a 550-km, $8^{\circ}$ inclination orbit indicate sensitivities for a $3 \%$ broadened $847 \mathrm{keV}$ line of $1.2 \times 10^{-6} \mathrm{ph} \mathrm{cm}^{-2} \mathrm{~s}^{-1}\left(10^{6} \mathrm{~s}\right.$, on-axis)-enabling ACT to achieve its primary science goal of systematically studying ${ }^{56} \mathrm{Co}$ emission from SN Ia. In the process, ACT becomes a powerful instrument for all classes of $\gamma$-ray observations (Table 2), including diffuse nuclear lines, compact objects, and GRBs. The simulations behind these results are discussed in more detail elsewhere [45]. Narrow-line sensitivity and angular resolution of the optimized S-Ge baseline instrument are presented here (Fig. 5). 

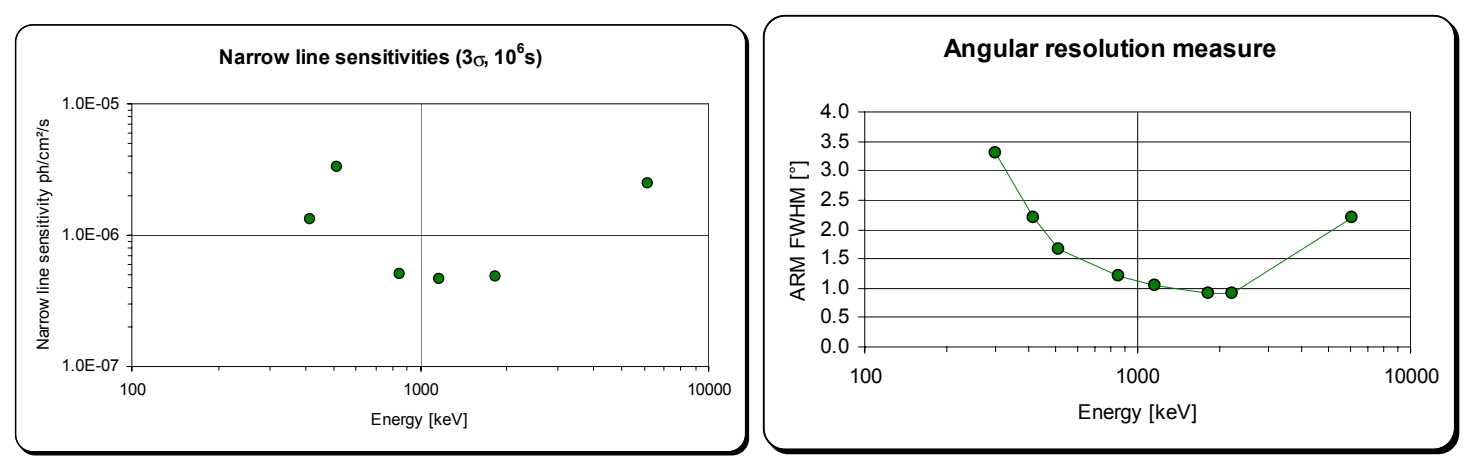

Fig. 5. (Left) Narrow-line, on-axis point-source sensitivity as function of energy. Sensitivities over the main nuclear range, $0.8-2 \mathrm{MeV}$, are $100 \times$ better than COMPTEL, 50× INTEGRAL/SPI. The instrument is sensitive at least down to the r-process-induced line at $415 \mathrm{keV}$ and up to cosmic-ray induced lines at $6 \mathrm{MeV}$. (Right) Instrument on-axis angular resolution. Combined with its high sensitivity, ACT will start resolving regions of Galactic nucleosynthesis. GRBs and other strong point sources can be localized to 5 '.

\section{MISSION OVERVIEW}

The ACT mission consists of a single instrument composed of a large array of position-sensitive detectors, surrounded by anti-coincidence shields and mounted on a zenith-pointing spacecraft (S/C) (Fig. 6). From the mission perspective (Table 3), ACT could be launched as early as 2015 from Kennedy Space Center (KSC) on a Delta IV 4240 vehicle. The Delta IV 4240 can deploy ACT into its baseline $550-\mathrm{km}, 8^{\circ}$ inclination circular orbit. A 5 -year minimum (10-year desired) lifetime is required to meet the primary science goals of the mission. Launch later than 2015 may require a slightly higher orbit, depending on the phase of the solar cycle.

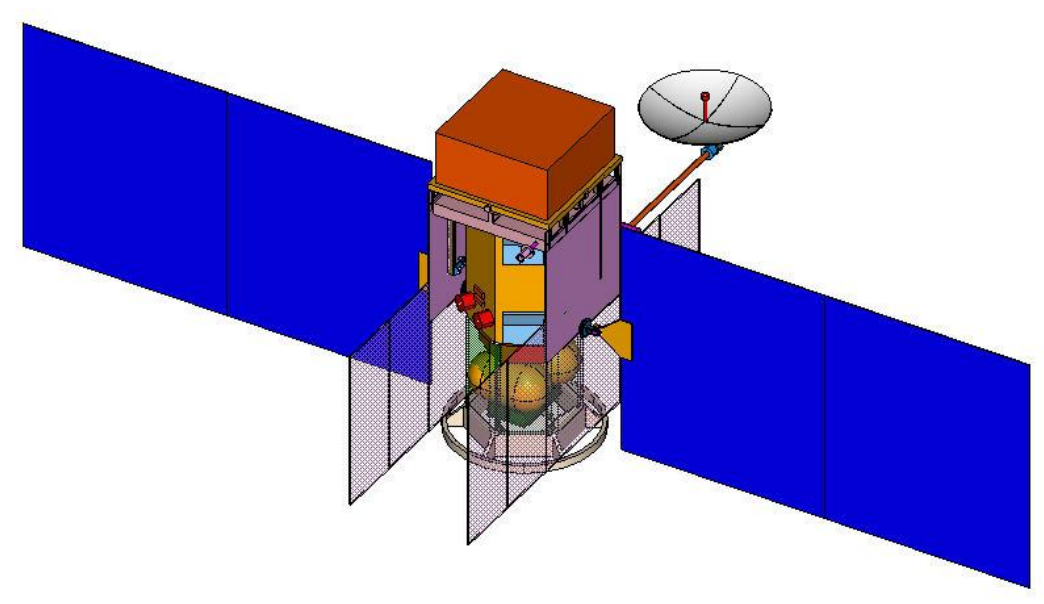

Fig. 6. ACT science instrument on its spacecraft bus, with solar panels, thermal radiators (transparent), and high-gain TDRSS antenna deployed.

ACT is a wide FoV instrument ( $25 \%$ of sky), surveying the entire sky by maintaining a zenith-pointed orientation and sweeping out the sky over the course of its orbit. Pointing attitude $\left( \pm 1^{\circ}\right)$ and aspect $\left( \pm 1^{\prime}\right)$ are fairly relaxed. In normal operational mode, the detectors must be cooled while observations are conducted. A low-inclination orbit will allow the instrument to operate with a nearly $100 \%$ duty cycle while remaining out of the South Atlantic Anomaly (SAA) with its intense radiation. All photon data will be passed through a simple filtering routine in the science instrument processor to identify candidate events. For the selected events, energy, position, and timing data, together with instrument aspect data, will be packaged and stored in the S/C 100-Gbyte solid-state data recorder for telemetry to the ground. 
Table 3. ACT mission requirements.

\begin{tabular}{|l|}
\hline Launch $\sim 2015,5-10$ year lifetime \\
\hline $550 \mathrm{~km} \mathrm{LEO},<10^{\circ}$ inclination, Delta IV (4240) \\
\hline $1^{\circ}$ attitude, $1^{\prime}$ aspect, zenith pointer \\
\hline Instrument $2100 \mathrm{~kg}$, S/C $1425 \mathrm{~kg}$, propellant $462 \mathrm{~kg}$ \\
\hline $3340 \mathrm{~W}$ power, $69 \mathrm{Mbps}$ telemetry \\
\hline \$760M (FY04, original IMDC estimate) \\
\hline
\end{tabular}

Data will be telemetered through TDRSS to White Sands and then transferred to the ACT Mission Operations and Distribution Center at the Goddard Space Flight Center for zero-level processing. The instrument data and required S/C data will be transferred to the Science Operations Center (SOC) located at the PI institution or GSFC. The SOC will make these data and analysis software available on-line to the investigator community after initial processing. Final archiving of the data and analysis software will be at the NASA High Energy Astrophysics Science Archive Research Center (HEASARC).

In addition, data are processed in real-time in the on-board science processor to detect and localize gamma-ray bursts and other transient events. Within tens of seconds, the transient's coordinates will be sent through a dedicated TDRSS SBand transmitter to the ground to enable prompt multi-wavelength observations.

The mass constraints on the mission come from the baseline launch vehicle, a Delta 4240 ( $4772 \mathrm{~kg}$ to $550 \mathrm{~km} / 8^{\circ}$ orbit). An instrument of $2100 \mathrm{~kg}$ and $2500 \mathrm{~W}$ power requirement (contingency included) could be launched as part of a S/C with total (dry) mass $4062 \mathrm{~kg}$, total power $3.34 \mathrm{~kW}$ (both include margins), and TDRSS-supported Ku-band communications to achieve the ACT objectives. All S/C bus components were found to be TRL 7-9 for a launch in 2015. (The mass and power envelopes for the Delta 4240 launcher and IMDC S/C were used to provide a design envelope for the different alternative ACT instrument designs considered as part of the ACT study.)

\section{TECHNOLOGY RECOMMENDATIONS}

During the course of the IMDC studies, it became clear to the team members that ACT, while a challenging mission, is not constrained by, or placing new requirements on, space mission architecture. All the mission technologies are either flight ready or soon forthcoming (TRL 7-9 for 2015 launch). The primary hurdles in the flight of ACT are in developing the enabling technologies for the science instrument itself. Even here, the prospects for ACT are encouraging because most of the technologies have been demonstrated either in flight or in the laboratory. The primary challenges arise from the scale of ACT. Technologies that have been demonstrated and/or flown for smaller instruments create challenges for ACT in terms of dead time, power, complexity, reliability, telemetry, backgrounds, and ultimately sensitivity.

The primary recommendations for ACT enabling technologies (more details in Ref. [48]), in support of the primary $\mathrm{SNe}$ Ia science goals, are:

1. Ge Detectors: enabling technology development, including electrode optimization, environmental testing, and manufacturing of large numbers

2. Thick Si Detectors: basic detector development for thicker detectors, plus enabling technology developments for manufacturing of large numbers

3. LXe Detectors: basic laboratory performance demonstration of optimized spectral performance

4. Readout ASICs: development of low power readouts, approaching $1 \mathrm{~mW} / \mathrm{channel}$ and $0.1 \mathrm{~mW}$ FETs

5. Cryogenics: detailed technical study, and enabling development of scaled-up systems

6. Structural Materials: engineering studies and demonstration of low-mass, low-Z support structures and cryostats

7. Simulation Toolset: long term investment in ground-up development of an integrated simulation package, including tested environmental inputs, faster processing, and novel data analysis and imaging techniques 
In addition, to validate the performance of the ACT baseline instrument, we recommend that a subscale prototype, incorporating the primary flight technologies in the flight configuration, be flown on a high altitude balloon flight for verification of both the instrument technologies and the simulation tools in a space environment.

\section{SUMMARY}

The Advanced Compton Telescope (ACT) will be a powerful new survey instrument for studying supernovae, Galactic nucleosynthesis, GRBs, compact objects, and fundamental physics. The ACT instrument design is driven by its primary

science goal: spectroscopy of the ${ }^{56} \mathrm{Co}(0.847 \mathrm{MeV})$ line from SNe Ia, which we expect to be broadened to $\sim 3 \%$. During the course of this study, we achieved a baseline instrument and mission design which will allow ACT to achieve its primary science goals, with a launch as soon as 2015. ACT will allow hundreds of SNe Ia detections over its primary 5year survey lifetime. In the process, ACT becomes a powerful all-sky observatory for all classes of $\gamma$-ray observations.

\section{ACKNOWLEDGEMENTS}

This research was supported by NASA grant NNG04GK30G.

\section{REFERENCES}

1. Ahn, K., et al., "Cosmic gamma-ray background from type Ia supernovae reexamined: Evidence for missing gamma rays at MeV energy," Phys. Rev. D 71, 121301 (2005).

2. Aprile, E., et al., "Spectroscopy and imaging performance of the Liquid Xenon Gamma-Ray Imaging Telescope (LXeGRIT)," SPIE 4140, 333 (2000).

3. Aprile, E., et al., "A liquid xenon time projection chamber for gamma-ray imaging in astrophysics: present status and future directions," NIM A461, 256 (2001).

4. Aprile, E., et al., "LXeGRIT Compton telescope prototype: current status and future prospects," SPIE 4851, 1196 (2003).

5. Benetti, S., et al, "Supernova 2002bo: inadequacy of the single parameter description," MNRAS 348, 261(2004).

6. Black, J.K., et al., "Imaging microwell detectors for x-ray and gamma-ray applications," SPIE 4140, 313 (2000).

7. Boggs, S. E., et al., "Upcoming balloon flight of the nuclear Compton telescope," SPIE 4851, 1221 (2003).

8. Branch, D., et al., "In search of the progenitors of Type IA supernovae," PASP 107 (1995).

9. Clayton, D.D., et al., "Gamma-ray lines from young supernova remnants," ApJ 155, 75 (1969).

10. Clayton, D.D, and Silk, J., "Measuring the rate of nucleosynthesis with a gamma-ray detector," ApJ 158, L43(1969).

11. Coburn, W., et al., "3D positioning germanium detectors for gamma-ray astronomy," SPIE 4784, 54 (2002).

12. Deines-Jones, P., et al., "Imaging micro-well proportional counters fabricated with masked UV laser ablation," NIM A477, 55 (2002).

13. Deines-Jones, P., et al., "Large-area imaging micro-well detectors for high-energy astrophysics," NIM A478, 130 (2002).

14. Dominguez, I., et al., "Evolution of type Ia supernovae on cosmological time scales," MmSAI 71, 449 (2000).

15. Georgii, R., et al., "COMPTEL upper limits for the 56Co gamma -ray emission from SN1998bu," $A \& A$ 394, 517 (2002).

16. Hillebrandt, W., and Niemeyer, J.C., "Type IA supernova explosion models," ARA\&A 38, 191 (2000).

17. Hoeflich, P., and Khokhlov, A., "Explosion models for Type IA supernovae: a comparison with observed light curves, distances, Ho, and Qo," ApJ 457, 500 (1996).

18. Hoeflich, P., et al., "Hard X-rays and gamma rays from type IA supernovae," ApJ 492, 228 (1998).

19. Kanbach, G., et al., "Development and calibration of the tracking Compton/Pair telescope MEGA," NIM A541, 310 (2005).

20. Kroeger, R.A., et al., "Position sensitive germanium detectors for the Advanced Compton Telescope," AIP Conf. Proc 510: Fifth Compton Symposium, 794, (2000).

21. Kurfess, J.D., et al., "Development and applications of position-sensitive solid-state gamma ray detectors," NIM A505, 256 (2003).

22. Leising, M., et al., "CGRO/OSSE observations of Supernova 1998bu," BAAS 31, 703 (1999).

23. Lichti, G.G., et al., "COMPTEL upper limits on gamma-ray line emission from Supernova 1991T," A\&A 292, 569 (1994). 
24. Lin, R.P., et al., "The Reuven Ramaty High-Energy Solar Spectroscopic Imager (RHESSI)," Solar Physics 210, 33 (2002).

25. McConnell, M. L., et al., "The development of coplanar CZT strip detectors for gamma-ray astronomy," AIP Conf. Proc. 587: Gamma 2001, 909 (2001).

26. Michelson, P.F., "Instrumentation for the Gamma-ray Large Area Space Telescope (GLAST) mission," SPIE 4851, 1144 (2003).

27. Morris, D.J., et al., "Reassessment of the 56Co emission from SN 1991T," AIP Conf. Proc. 410: Fourth Compton Symposium, 1084 (1997).

28. Nomoto, K., et al., "Accreting white dwarf models of Type I supernovae. III - carbon deflagration supernovae," ApJ 286, 644 (1984).

29. O'Neill, T.J., et al., "The TIGRE gamma-ray telescope," AIP Conf. Proc. 587: Gamma 2001, 882 (2001).

30. Pelling, R. M., et al., "Spatial properties of large-area crossed-strip CZT detectors," SPIE 4784, 21 (2002).

31. Perlmutter, S., et al., "Measurements of the cosmological parameters Omega and Lambda from the first seven supernovae at $\mathrm{z}>=0.35, " \mathrm{ApJ} 483,565$ (1997).

32. Phillips, M.M., "The absolute magnitudes of Type IA supernovae," ApJ 413, L105 (1993).

33. Phlips, B.F., et al., "Development of thick intrinsic silicon detectors for hard X-ray and gamma-ray detection," IEEE NSS Conf. Rec. 1, 207 (2001).

34. Riess, A.G., et al., "Observational evidence from supernovae for an accelerating universe and a cosmological constant," AJ 116, 1009 (1998).

35. Rudy, R.J., et al., "Early-time infrared spectra of the Type Ia Supernova 2000cx," ApJ 565, 413 (2002).

36. Ruiz-Lapuente, P., et al., "The cosmic gamma-ray background in the MEV range," ApJ 549, 483 (2001).

37. Ryan, J.M., et al., "Development of CZT strip detector modules for 0.05- to 1-MeV gamma-ray imaging and spectroscopy," SPIE 4851, 885 (2003).

38. Schönfelder, V., et al., "Instrument description and performance of the Imaging Gamma-Ray Telescope COMPTEL aboard the Compton Gamma-Ray Observatory," ApJS 86, 657 (1993).

39. Shah, K.S., et al., "LaBr/sub 3/:Ce scintillators for gamma-ray spectroscopy," IEEE TNS 50, 2410 (2003).

40. Strigari, L.E., et al., "The concordance cosmic star formation rate: implications from and for the supernova neutrino and gamma ray backgrounds," JCAP 0504, 017 (2005).

41. Vedrenne, G., et al., "SPI: The spectrometer aboard INTEGRAL," $A \& A$ 411, L63 (2003).

42. Watanabe, K., et al., "The diffuse gamma-ray background from supernovae," ApJ 516, 285 (1999).

43. Watanabe, K., et al., "The MeV cosmic gamma-ray background measured with SMM," AIP Conf. Proc. 510: Fifth Compton Symposium, 471 (2000).

44. Weidenspointner, G., et al., "The cosmic diffuse gamma-ray background measured with COMPTEL," AIP Conf. Proc. 510: Fifth Compton Symposium, 467 (2000).

45. Wunderer, C., et al., "The ACT vision mission study simulation effort," New Astr. Rev., (in press) (2006).

46. Zhang, P., and Beacom, J.F., "Angular correlations of the MeV cosmic gamma-ray background," ApJ 614, 37 (2004).

47. http://cryowwwebber.gsfc.nasa.gov

48. Boggs, S. E., et al., "The Advanced Compton Telescope mission,“ AIAA, (in press) (2006). 